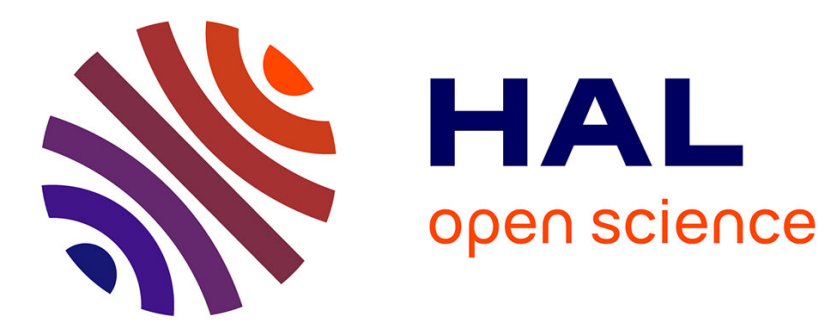

\title{
Assessment of the influence of the distance between two wave energy converters on the energy production
}

\author{
A. Babarit, B. Borgarino, P. Ferrant, A.H. Clement
}

\section{To cite this version:}

A. Babarit, B. Borgarino, P. Ferrant, A.H. Clement. Assessment of the influence of the distance between two wave energy converters on the energy production. IET Renewable Power Generation, 2010, 4 (6), pp.592-601. 10.1049/iet-rpg.2009.0190 . hal-01145133

\section{HAL Id: hal-01145133 \\ https://hal.science/hal-01145133}

Submitted on 15 Mar 2019

HAL is a multi-disciplinary open access archive for the deposit and dissemination of scientific research documents, whether they are published or not. The documents may come from teaching and research institutions in France or abroad, or from public or private research centers.
L'archive ouverte pluridisciplinaire HAL, est destinée au dépôt et à la diffusion de documents scientifiques de niveau recherche, publiés ou non, émanant des établissements d'enseignement et de recherche français ou étrangers, des laboratoires publics ou privés. 


\title{
Assessment of the influence of the distance between two wave energy converters on the energy production
}

\author{
Aurélien Babarit, Bruno Borgarino, Pierre Ferrant, Alain Clément ${ }^{1}$ \\ ${ }^{1}$ Laboratoire de Mécanique des Fluides, CNRS UMR6598, Ecole Centrale de Nantes, 1, rue de la Noë, 44300 NANTES
}

\begin{abstract}
In this paper, an array of two interacting wave energy converters with hydraulic PTOs is considered. A time domain model is derived by using classical linear potential theory. Numerical simulations and parametric studies are performed in order to determine the influence of the distance on the capture width of each system in the array, both in regular and irregular waves. It is shown that when the systems are close, the front system (which meets the waves first) is more affected by the wave interaction than the rear system. But the wave interactions decrease faster with the distance for the front system than for the rear system. For this latter system, it seems that the effect of wave interactions remains noticeable even far (14 diameters) from the first system.
\end{abstract}

Keywords: Wave Energy Converters, Array, Wave interactions, Numerical simulation

\section{Introduction}

Like wind turbines, wave energy converters are designed to be deployed in large arrays composed of many units. Each unit is going to interact with all the others, by absorbing, radiating and diffracting the waves. The spatial scattering of the units in the array and the interactions between them can have a positive (focusing of the waves, smoothing of the output power [1], [2]) or a negative effect (masking effects), depending on the configuration of the array. Therefore, it is of great interest to be able to predict these interactions, in order to use them beneficially or to avoid them. This has been a subject of investigations by several R\& D units across Europe in the past, see the pionner work of [3], [4], [5], [6], [7], [8], and it is still an area of research, as several studies which have been published recently show, see [9],[10], [11], [12].

However, it is well known that the far field of radiated and diffracted waves decay with the square-root of the distance to the body which generated them. Hence, one can assume that when the systems are sufficiently far from each other, these interactions should become negligible. So, the question is : how far is sufficiently far?
This is the issue we tried to address in this paper, by assessing the influence of the distance between two wave energy converters on the energy production. As it depends on the considered wave energy converters, we first consider the case of two generic wave energy converters, i.e two cylindrical surface piercing heaving buoys with hydraulic PTOs. A hydraulic PTO induces nonlinearities in the system, as thus is better modelled in the time domain. Recent developments in our in house ACHIL3D numerical code gave us such ability, and comparisons of time domain simulations of the motion of the buoys are presented in regular and irregular waves as a function of the distance between the buoys. Finally, we considered the case of the SEAREV wave energy converter.

\section{Methods}

\subsection{Equation of motion of two interacting heaving wave energy converters}

Let us consider a basic array of wave energy converters, composed of two identical semi submerged cylinders and hydraulic PTOs, see figure (1). The diameter of each cylinder is taken equal to $10 \mathrm{~m}$ and their draught is equal to $10 \mathrm{~m}$. We assume that both cylinders can move only in the heave motion (i.e. along the vertical axis), with all other degrees of freedom ideally restricted. For each system, a hydraulic PTO composed of one hydraulic ram pumps oil from a Low Pressure (LP) tank to a High Pressure (HP) tank. For simplicity reasons, we considered that input and output flows from the hydraulic cylinders are mutualised in the same ducts and that the volumes of the HP and LP tanks are sufficiently large to consider that the low pressure $\left(p_{0}\right)$ and the high pressure $(p)$ in the hydraulic circuit remain constant.

Let us note with index 1 and 2 all quantities related respectively to the first and with the second system. Let $z_{1}$ and $z_{2}$ be the heave motion of each buoy. Let $\mathbf{Z}=$ $\left(\begin{array}{ll}z_{1} & z_{2}\end{array}\right)^{t}$ be the position vector of the whole array. Assuming the fluid to be non-viscid and incompressible, the flow to be irrotationnal and the amplitude of motions and waves to be sufficiently small in comparison with the wavelength, the classical linearized potential theory can be used as a framework for calculation of the fluidstructure interactions. Hence, one can write the equation of motion of the WEC in the time domain as: 


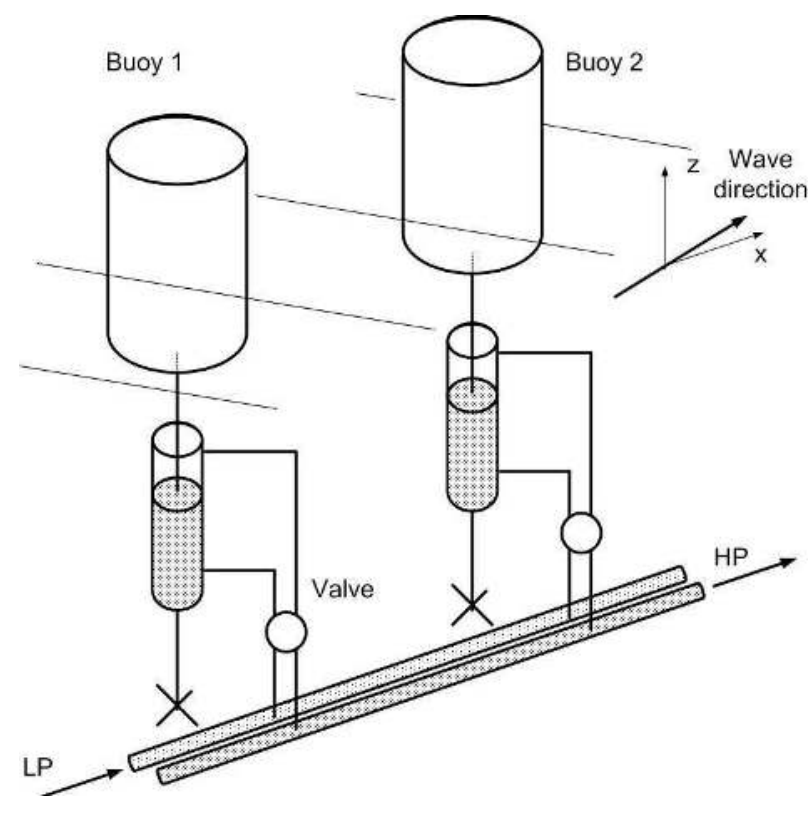

Figure 1: Schematic representation of a basic array of wave energy converters with hydraulic PTOs.

$$
\begin{array}{r}
\left(\mathbf{M}+\left[\mu_{\infty}\right]\right) \ddot{\mathbf{Z}}+\int_{0}^{t} \mathbf{K}_{\mathrm{rad}}(t-\tau) \dot{\mathbf{Z}}(\tau) d \tau+\left(\mathbf{K}_{H}+\mathbf{K}_{A}\right) \mathbf{Z} \\
=\mathbf{F}_{e x}+\mathbf{F}_{P T O}
\end{array}
$$

with:

- $\dot{\mathbf{Z}}, \ddot{\mathbf{Z}}$ being respectively the velocity and acceleration vectors of the WECs.

- $\mathbf{M}=\left(\begin{array}{cc}m_{1} & 0 \\ 0 & m_{2}\end{array}\right)$ the mass matrix of the system. As we considered identical cylinders, $m_{1}=m_{2}=$ $785 \mathrm{t}$.

- $\mathbf{K}_{H}=\left(\begin{array}{cc}k h_{1} & 0 \\ 0 & k h_{2}\end{array}\right)$ the hydrostatic stiffness matrix of the system. Again, $k h_{1}=k h_{2}=770 \mathrm{kN} . \mathrm{m}^{-1}$.

- $\mathbf{K}_{A}$ an additional stiffness matrix which represents the action of possible moorings. In this study, it was neglected, i.e. $\mathbf{K}_{A}=\mathbf{0}$

- $\left[\mu_{\infty}\right]=\left(\begin{array}{cc}\mu_{1} & \mu_{12} \\ \mu_{21} & \mu_{2}\end{array}\right)$ the added mass matrix and $\mathbf{K}_{\mathbf{r a d}}=\left(\begin{array}{cc}k r_{1} & k r_{12} \\ k r_{21} & k r_{2}\end{array}\right)$ the radiation impulse response matrix which represents the radiation of waves by the body after an impulsive velocity at $t=0$, according to the classical Cummins' decomposition [13]. In these matrices, the non diagonal terms are not anymore equal to 0 . Indeed, they represent the pressure force measured on the second body due to the radiated wave associated with an impulsive velocity applied to the first one. For obvious symmetry reasons, we have here $\mu_{1}=\mu_{2}$, $\mu_{12}=\mu_{21}, k r_{1}(t)=k r_{2}(t), k r_{12}(t)=k r_{21}(t)$.
- $\mathbf{F}_{e x}$ is the excitation vector, associated to the action of incident and diffracted wave fields upon the WECs. Using King's approach [14], let $\mathbf{K}_{e x}(t, \beta)$ be the force response associated to an impulsive elevation on the free surface propagating along a direction such as the angle between this direction and the $\mathrm{x}$ axis is $\beta$. Using the superposition principle, owing to the global linearity of the problem solved here, the generalized excitation force is then given by

$$
\mathbf{F}_{e x}(t)=\sum_{\beta} \int_{0}^{t} \mathbf{K}_{e x}(t-\tau, \beta) \eta(\tau, \beta) d \tau
$$

with $\eta(t, \beta)$ being the component of the free surface elevation at a given reference location propagating in the direction $\beta$. In case of a regular wave, $\eta(t, \beta)$ is a simple sine function $a \sin (\omega t+\varphi)$ with $a$ the amplitude of the wave, $\omega$ its circular frequency and $\varphi$ an initial phase. In case of random waves, $\eta(t, \beta)$ will be considered here as a sum of $N_{c}$ elementary sine functions whose amplitudes $\left(a_{j}\right)_{j=1, N_{c}}$ are derived from the standard Jonswap energy spectrum [15] using the methodology preconized by Ricci et al. in [16]; and whose phases $\left(\varphi_{j}\right)_{j=1, N_{c}}$ are set randomly.

- $\mathbf{F}_{\text {PTO }}$ is the force vector associated to the action of the PTOs. If the PTOs are linear dampers, $\mathbf{F}_{\text {PTO }}$ is given by :

$$
\mathbf{F}_{\text {PTO }}=\mathbf{B}_{\text {PTO }} \dot{\mathbf{Z}}
$$

with $\mathbf{B}_{\text {PTO }}$ being the PTO damping coefficient matrix. In this study, we considered hydraulic PTOs. Hence, the PTO force is given by :

$$
\mathbf{F}_{P T O}=-\left(p-p_{0}\right) S \cdot \operatorname{sign}(\dot{\mathbf{Z}})
$$

\subsection{Numerical model}

A numerical model was written in Fortran in order to solve equation (1). The hydrodynamic coefficients and functions were calculated using the BEM based code ACHIL3D [17], which was recently extended in order to be able to deal with several independent floating bodies [18]. The Impulse Response Functions (IRF) of the radiation forces and excitation forces are plotted in figure (2) in the case of the two cylinders separated by a distance of 2 diameters. In this example, the IRF of the excitation forces is associated with an impulse on the free surface located at the middle of the two cylinders and which propagates along the $\mathrm{x}$ axis. One could notice that the amplitude of the crossed IRF $k_{12}$ of the radiation force is the same order than $k_{1}$, which shows a strong interactions between the buoys in the velocity part of the radiation force. However, the calculated values of the added masses are $\mu_{1}=\mu_{2}=250 \mathrm{t}$ and $\mu_{12}=\mu_{21}=11.5$ $\mathrm{t}$, which does not show the same strong interaction for the acceleration part.

As an illustration, figure (3) shows time series of the heave motion and of the output flow of the two cylinders 

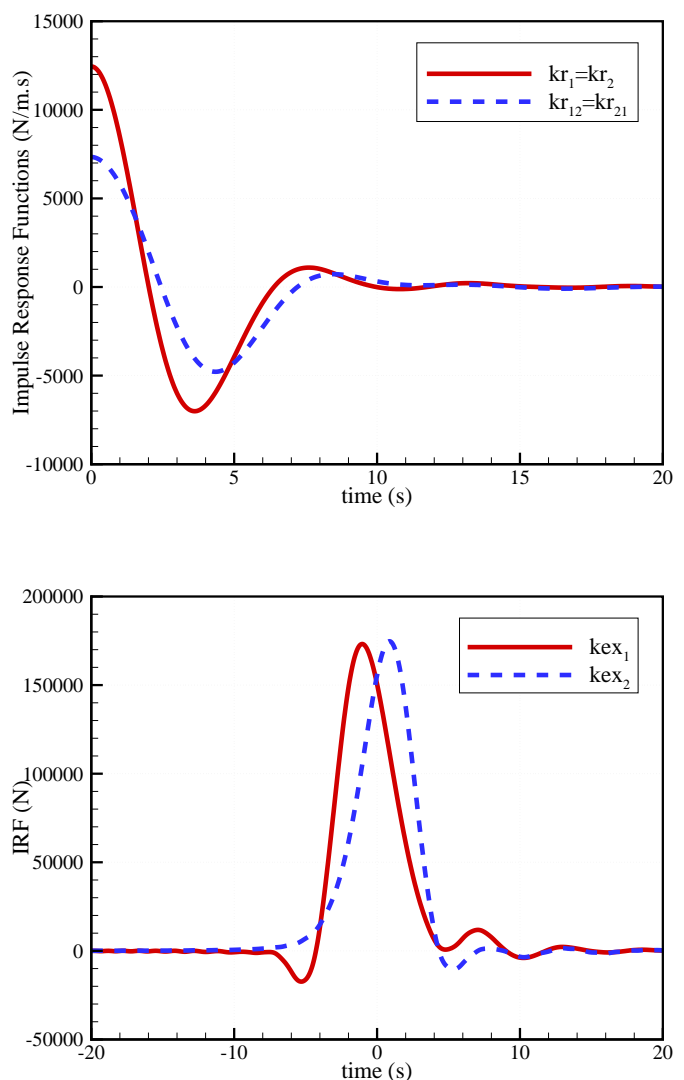

Figure 2: Impulse Response Functions (IRFs)of the radiation and excitation forces in heave in the case of two cylinders separated by a distance of $20 \mathrm{~m}$. One could notice that the amplitude of the crossed IRF $k_{12}$ of the radiation force is the same order as $k_{1}$.

calculated with this model in response to a monodirectionnal irregular wave, with a peak period $T_{p}$ equal to $10 \mathrm{~s}$, a significant height $H_{s}$ equal to $2 \mathrm{~m}$, a frequency spreading equal to $\gamma=3.3$ and $\beta=0^{\circ}$. The pressure difference in the hydraulic circuit was chosen equal to $p-p_{0}=20$ bars and the area of the hydraulic cylinders $S=706 \mathrm{~cm}^{2}$.

In this figure, one can observe that the overall behaviour of the responses of the two systems are similar, but with a phase shift due to their spatial scattering. One can also observe that there are some moments during when the buoys appear to be latched. This is due to the fact that with hydraulic PTOs, the PTO force is a Coulomb damping which can exceed the hydrodynamic force. Hence, if the pressure difference is too high in the hydraulic circuit, it can prevent any motion in the system. These effects were observed by [19] on a generic heaving WEC and by [20] on the SEAREV WEC. It was even used by Falcao in [21] to phase control the motion of a generic heaving WEC by tuning the hydraulic circuit parameters to the sea state, but this was not tried in this study.
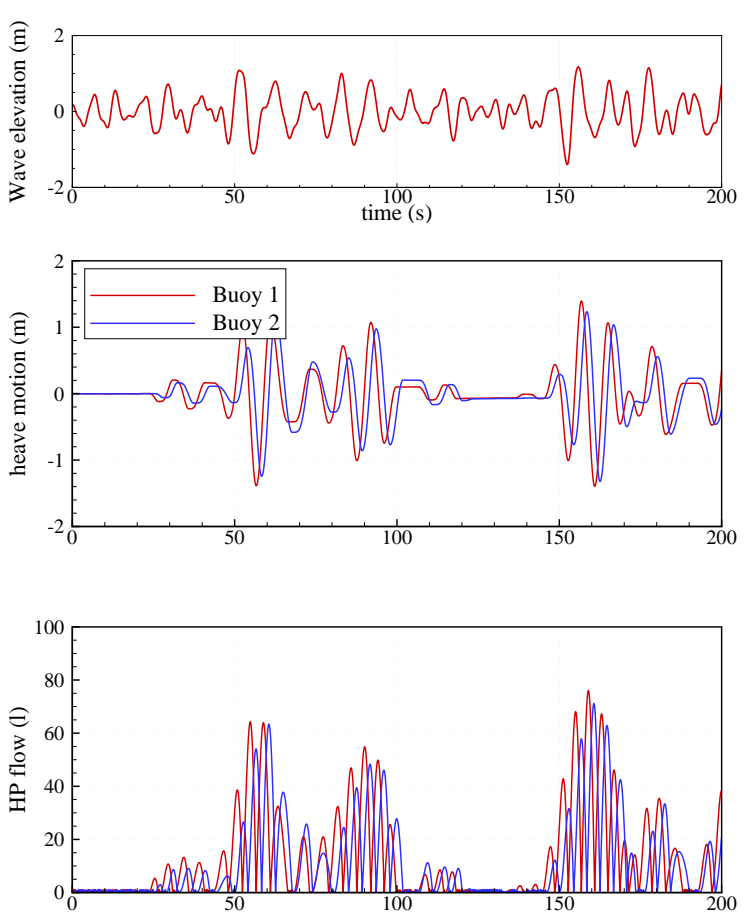

Figure 3: Time series of the free surface elevation (top), of the heave motion (middle) and of HP flow (bottom) of two generic wave energy converters. Characteristics of the monodirectional incident wave are $T_{p}=10 \mathrm{~s}, H_{s}=2 \mathrm{~m}$ and $\gamma=3.3$. The distance between the two cylinders is $20 \mathrm{~m}$.

\subsection{Layout of the array}

We considered the layout shown in figure (4) for the array. There are two main parameters, which are the distance $d$ between the two systems and the main direction of propagation of the incident waves $\beta$. In this study, we considered only waves propagating in the direction $\beta=0$.

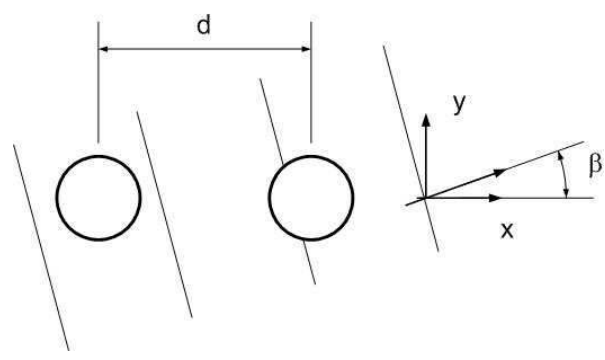

Figure 4: Layout of the considered array. Main parameters are the distance $d$ between the two systems and the main direction of propagation of the waves $\beta$.

\section{Results}

\subsection{Regular waves}

As we considered hydraulic PTOs, parameters of the hydraulic circuit need to be controlled and optimised in order to adapt the PTO force to the level of wave energy in each state. There are many ways of doing so 

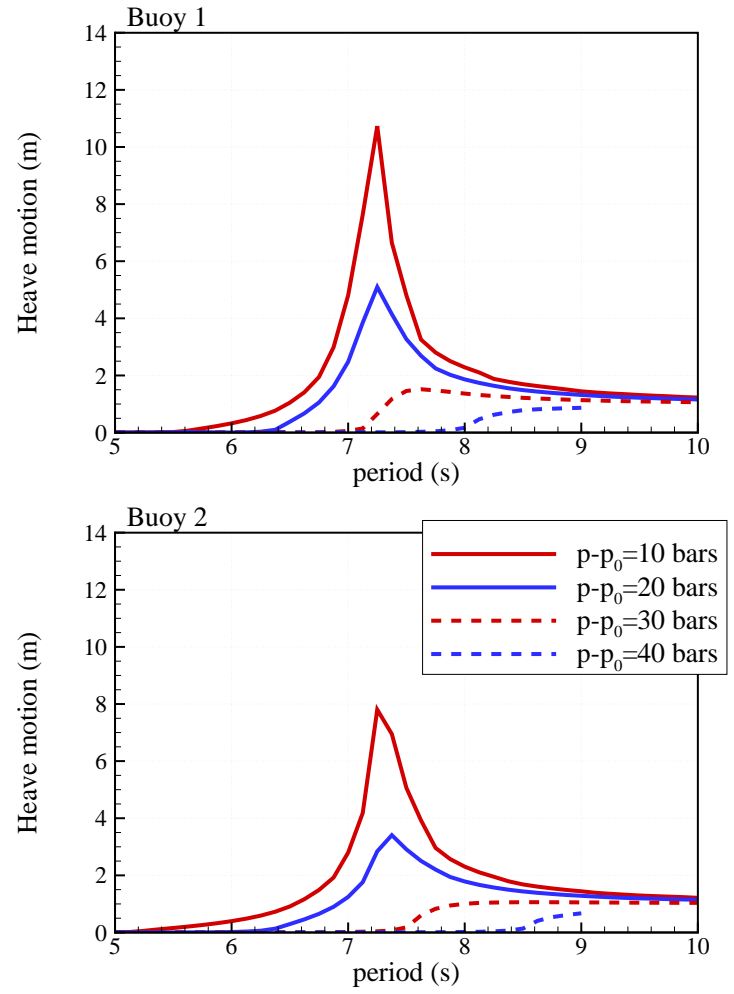
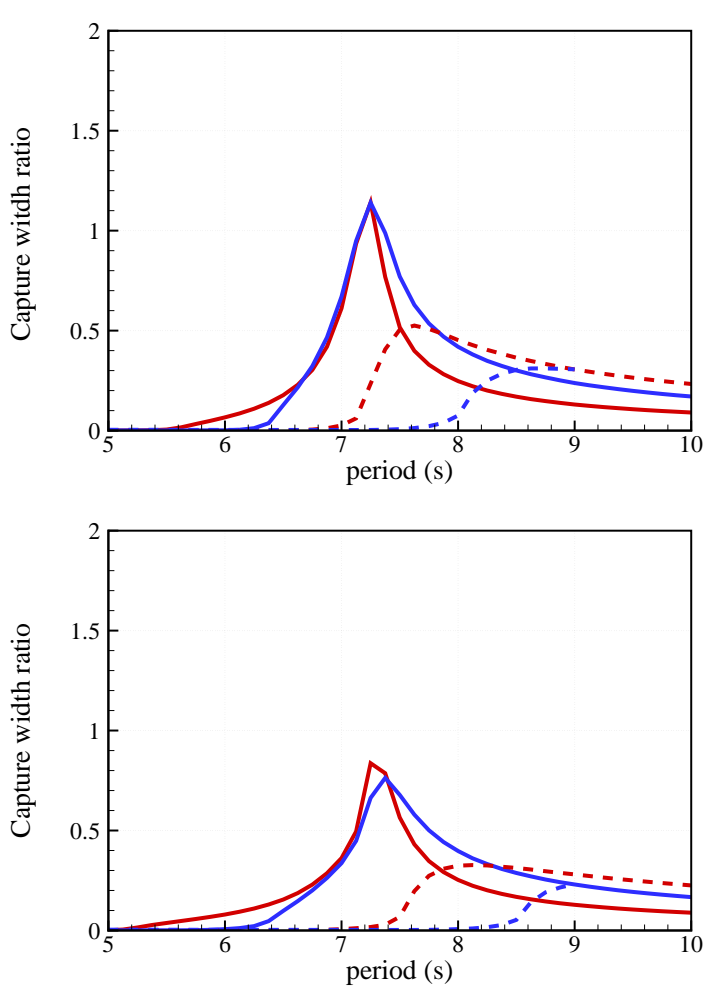

Figure 5: Transfer functions and capture width ratio of the first system (top figure) and of the second system (bottom figure) as a function of pressure difference in the hydraulic circuit.

and one can refer to [22] for an exhaustive review of all of them. However, in this study, we preferred doing parametric studies of the influence of the pressure diffence $\Delta p=\left(p-p_{0}\right)$ in the hydraulic circuit (which is the only PTO parameter in the model we considered) instead of choosing a particular control for the PTO force. From the energy production point of view, this is not a very satisfactory approach as an adequate control could probably be able to improve the power absorption, but it was found to be sufficient regarding the aim of the paper, which is to to assess the influence of the distance between two systems on the energy production.

Figure (5) shows the results of such a parametric study. Four pressure differences were considered : $\Delta p=$ $10,20,30$ and 40 bars. The top (respectively bottom) figure shows the amplitude of the motion and the capture width ratio of the first (respectively second) system as a function of the wave period. The capture width ratio is defined as the ratio of the mean power extracted by the system from to the waves divided by the wave power available in a wave front the width $B$ of the device $P_{w}$. It is given by :

$$
P_{w}=B \frac{1}{8 \pi} \rho_{w} g^{2} a^{2} T_{w}
$$

where $\rho_{w}$ is the density of water, $g$ is the gravity, $a$ is the wave amplitude and $T_{w}$ is the wave period.

In this figure, each value of the mean absorbed power $\bar{P}_{i}$ is derived from the mean of the instanteous power $\left(P_{i}=\Delta p S\left|\dot{z}_{i}\right|\right)$ of the hydraulic cylinder over the last two thirds of a $400 \mathrm{~s}$ time domain simulation, in order to remove the effect of the transients. In these simulations, the wave amplitude was set equal to $1 \mathrm{~m}$, the wave direction is $\beta=0^{\circ}$ and the distance between the two buoys is $20 \mathrm{~m}$.

One can see that the optimal value of $\Delta p$ depends on the period of the wave. For short wave periods, a small $\Delta p$ gives higher capture width than large ones and for long wave periods, large pressure difference gives better energy production than small ones. However, one can see that the choice of the intermediate pressure difference of 20 bars is a good compromise, as it leads to the widest energy bandwidth, with, for almost all the wave periods considered, only a small reduction in the energy production in comparison with the maximum achievable with another pressure difference. Morevoer, it appears that, for the same capture width ratio, the amplitude of the motion at the natural period is reduced by a factor 2 , which leads to more realistic predicted amplitudes.

Figure (6) shows the capture width ratio of each system in the array as a function of the wave period. The same four pressure differences as before were considered and, for each pressure difference, we plotted the results for seven different distances $d$ between each system. For sake of comparison, we also plotted on each graph the capture width of a single isolated system. Notice that, in these simulations, the wave amplitude was set equal to 1 $\mathrm{m}$ and the wave direction is $\beta=0^{\circ}$.

In this figure, in a general manner, one can observe that the effect of the interactions on the capture width ratio are much stronger for the first body (which meets the wave first) than for the second one, especially when the two systems are close and when $\Delta p$ is large. This 

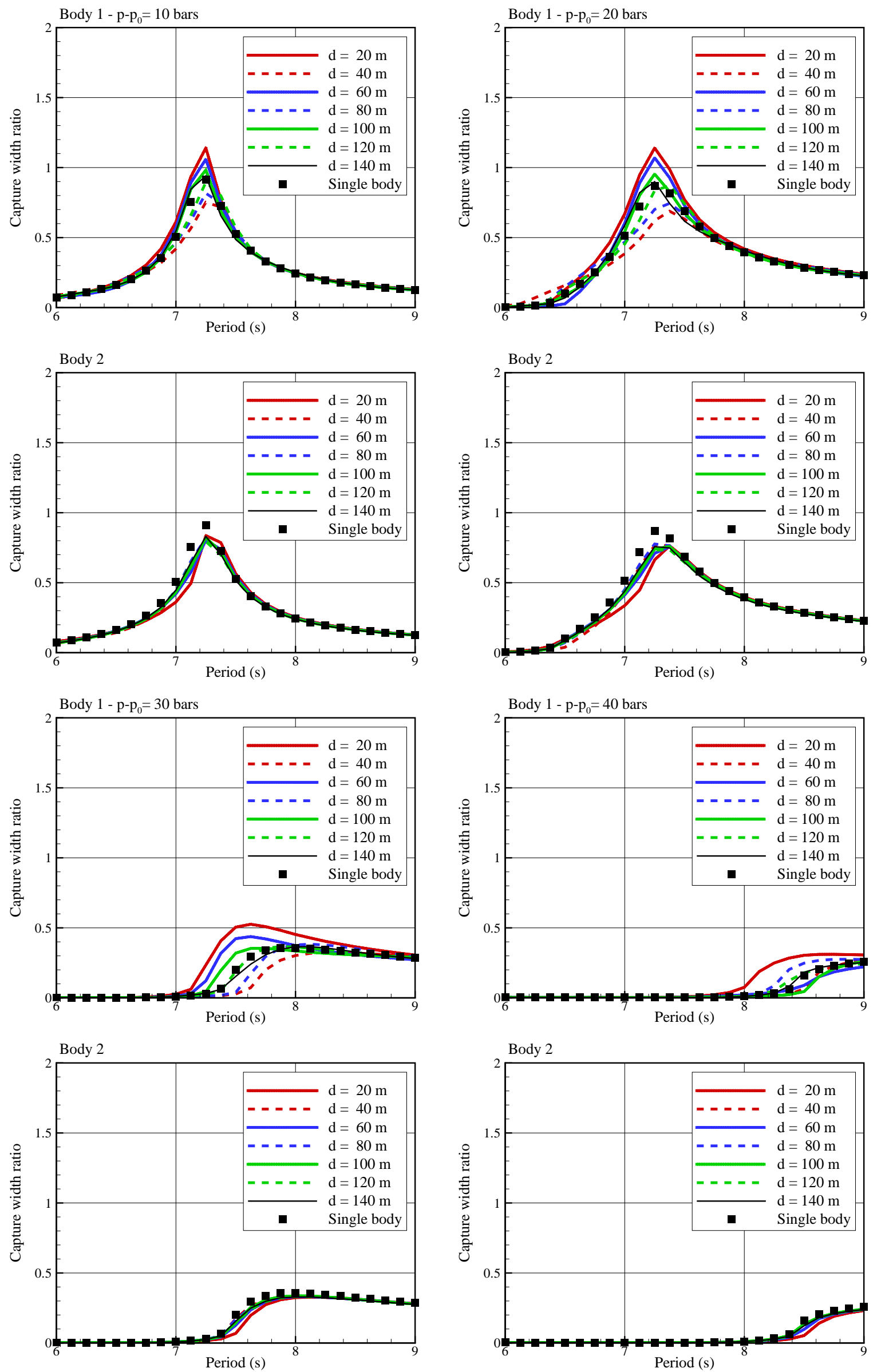

Figure 6: Capture width ratios of the first buoy (top figure) and of the second buoy (bottom figure) as a function of the pressure difference in the hydraulic circuit. 
is a quite surprising result, as one could have expected that the main effect would have been a reduction of the wave power in the wake of the second system and hence a decrease in the absorbed power from the rear system. Actually, it appears that it happens exactly the contrary, with only a slight reduction of the power function for the second system in comparison with the one of a single isolated system, and all the perturbations on the first one. One could notice that this result was already observed by Child et al. in [11] in the same case of two interacting cylinders.

Interactions between the two systems can lead to a higher or lower power absorption for the first system, depending both on the distance between the systems and on the pressure difference in the hydraulic circuit, but it seems that it does not depend on the wave period. So, one can define a criterion to get a synthetic view of the effect of the wave interaction on the power absorption. In this study, we defined the criterion $Q_{i}$ as the sum over the wave periods of the difference between the mean absorbed power $\bar{P}_{i}$ by the system $i$ and the mean absorbed power $\bar{P}_{\text {isolated }}$ by an isolated system divided by the sum of the mean absorbed power $\bar{P}_{\text {isolated }}$ :

$$
Q_{i}\left(T_{i}, T_{f}\right)=\frac{\int_{T_{i}}^{T_{f}}\left(\bar{P}_{i}(T)-\bar{P}_{\text {isolated }}(T)\right) d T}{\int_{T_{i}}^{T_{f}} \bar{P}_{\text {isolated }}(T) d T}
$$

In figure (7), we plotted these criteria $Q_{1}$ and $Q_{2}$, and also a criterion $Q$ defined as $Q=\frac{Q_{1}+Q_{2}}{2}$, as a function of the ratio $d / D$ of their distance divided by their diameters. It appears that :

- As one could have expected, the wave interactions are the strongest when the two systems are close. One can also see that the larger the pressure, the stronger the wave interactions effect is.

- The effect is not monotonous with the distance - as one can see on the first graph that each $20 \mathrm{~m}$ increase in the distance leads to the opposite effect than on the previous step on the first system - but it can probably be related with the ratio of the distance on the wavelength.

- The wave interactions decrease with the increasing distance between the systems. For the first system, one can see that it can be neglected when the two systems are separated by more than 10 diameters. However, for the second (rear) system, one can observe that the wave interactions effect remain significant for much longer distances. This could be explained, maybe, by the fact that some part of the incident wave energy has been absorbed by the first system, but this would require more investigations.

- For the whole array, wave interactions have a positive effect (more absorbed energy than two isolated systems) only in a few cases, mainly when the systems are close. Most of the time and particularly when the systems are well separated, wave interactions lead to a reduction of the absorbed energy in comparison with isolated systems. One could notice that the reduction is rather small (about 3\%), but that is for an array composed of only two systems. When the array is composed of more systems, one can expect that cumulative effects of wave energy reduction in the wake of front systems would lead to a higher absorbed energy reduction.

In order to determine the part from the radiated wave and the part from the diffracted wave in the effect of the wave interactions, we plotted on figure (8) a comparison of the capture width ratio of the first body when the second body is free and when it is fixed. In these simulations, $\Delta p=20$ bars and the wave amplitude was set equal to $1 \mathrm{~m}$.
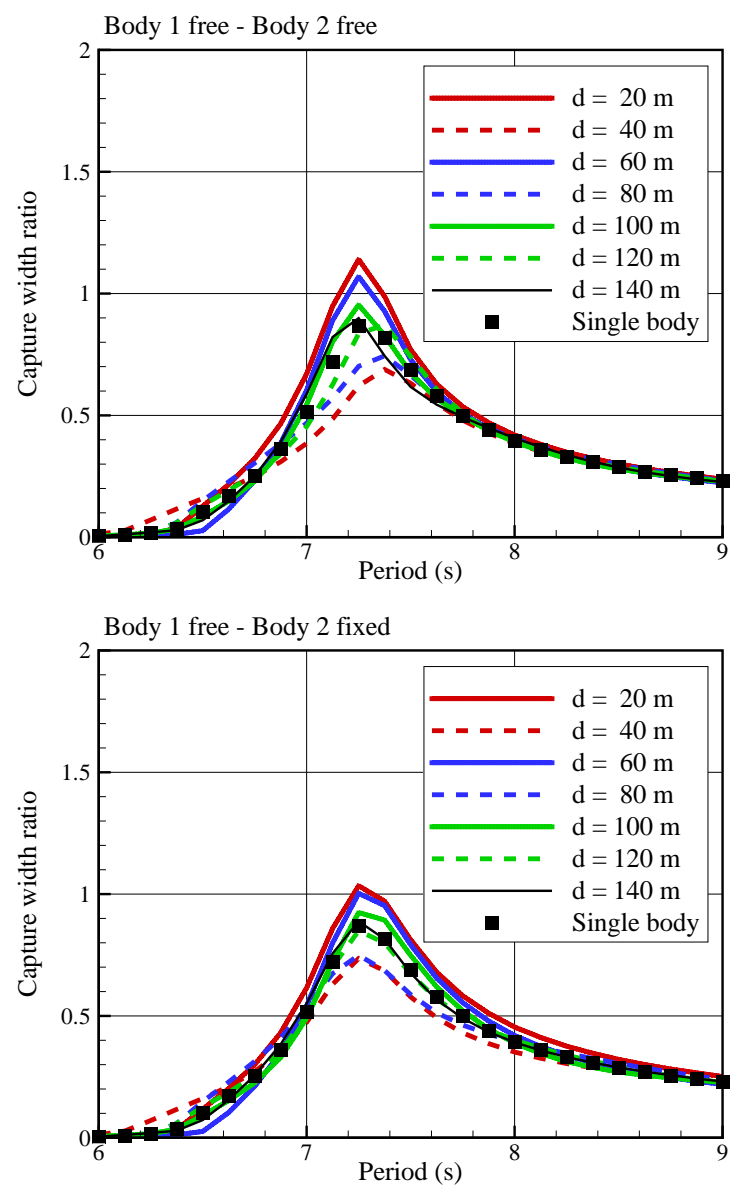

Figure 8: Capture width ratios of the first buoy when the second buoy is free (top figure) and when it is fixed (bottom figure).

One can observe that the effect of the wave interactions is reduced by a factor about two. Hence, it seems that radiation and diffraction contribute similarly to the modification of the capture width ratio.

\subsection{Irregular waves}

Figure (9) shows capture width ratios of the two interacting systems in irregular waves. The Jonswap spec- 
trum was used for the synthesis of irregular waves, with a significant height of $2 \mathrm{~m}$, a frequency spreading parameter set equal to $\gamma=3.3$, a wave angle equal to $\beta=0^{\circ}$ and a different set of phases for each simulation. Mean absorbed powers have been computed by averaging the mean absorbed power over five runs of $600 \mathrm{~s}$ time domain simulation. For sake of comparisons, we also plotted on this figure the capture width ratio of a single system.

Several distances between the two bodies were considered. The same conclusions as in regular waves can be observed from these figures, i.e

- The closer the two systems, the stronger the wave interactions effects are.

- Wave interactions can have a positive effect on the absorbed power for the first system. For the second one, it seems to be systematically negative.

- Taking into account the scattering effect on each plot of these graphs due to the random phases sets in irregular waves, it seems that the positiveness or negativeness effect of the wave interactions does not depend on the wave period.

In figure (10), we plotted the criteria $Q_{1}, Q_{2}$ and $Q$ derived from these simulations in order to determine the overall effect of the wave interactions. Again, one can observe that the results are very similar to those in regular waves : for the first system, the wave interactions decrease with the distance, and the energy absorption converges to the energy absorption of an isolated system whereas for the second system, the effect of the wave interactions is less oscillating and does not seem to vanish for the distances we considered. This leads to an about $4 \%$ overall reduction of the energy absorption of the array in comparison with the energy absorption of isolated systems.

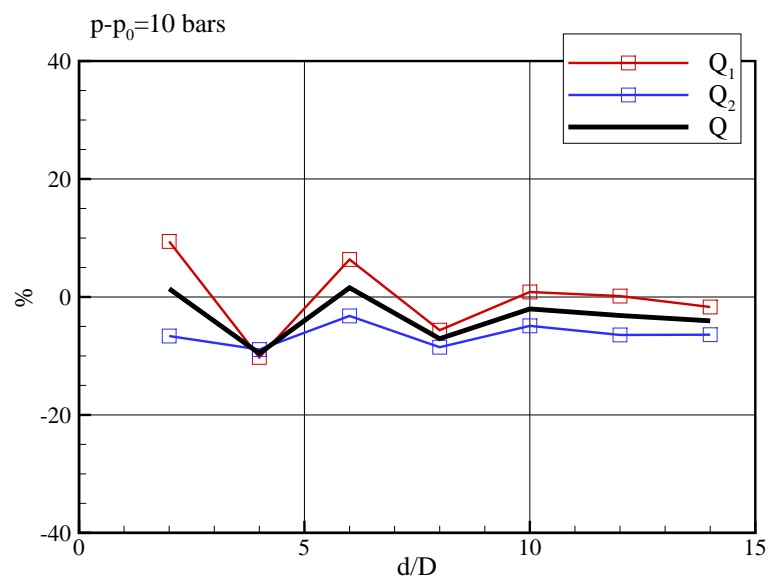

Figure 10: Perturbations of the energy absorption by wave interactions in an array of two systems as a function of their distance, in irregular waves.

\section{Application to the SEAREV device}

Unlike the systems previously studied, the SEAREV has a large width $(30 \mathrm{~m})$ facing the waves. Figure (11) shows the result of such calculations when the two systems are separated by a $40 \mathrm{~m}$ distance. For safety reasons such a configuration is unrealistic, but it shows interesting results for the understanding of interaction phenomenons.

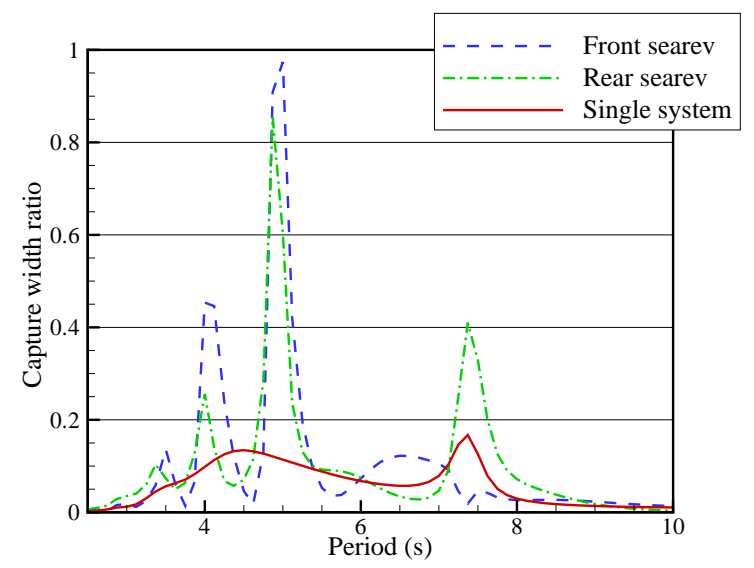

Figure 11: Capture width ratio of a 2-SEAREVs' array. The "front SEAREV" meets the wave first. For comparison, the capture width of a single SEAREV in the sea is also plotted.

The considered waves are regular, and their height has been set to $2 \mathrm{~m}$. As shown previously, the system which meets the wave first is the most influenced by interactions. The rear SEAREV (which meets the wave in second) has a power peak at the same wave period as a SEAREV alone, which is not the case for the front SEAREV(which meets the wave first). For both systems the shape of the capture width ratio is strongly modified by a peak around a $5 \mathrm{~s}$ wave period, which is a $40 \mathrm{~m}$ wave length, i.e the distance between the systems. This reveals a resonance effect : waves radiated and reflected between the two systems add their influences to incoming waves. With a higher distance between the SEAREVs, this effect would have probably been lower.

More calculations are currently carried on to determine the influence of interactions in a two SEAREV WECs' array. They will be presented at the conference.

\section{Conclusion}

In this paper, we considered the effect of wave interactions between two wave energy converters on their respective capture widths. It was observed that :

- The wave interactions are stronger when the systems are close.

- The strongest effects appear on the front system, but wave interactions effects decrease faster on this system than on the rear one. Contrarily to the front system, their effect is still noticeable on the rear system 

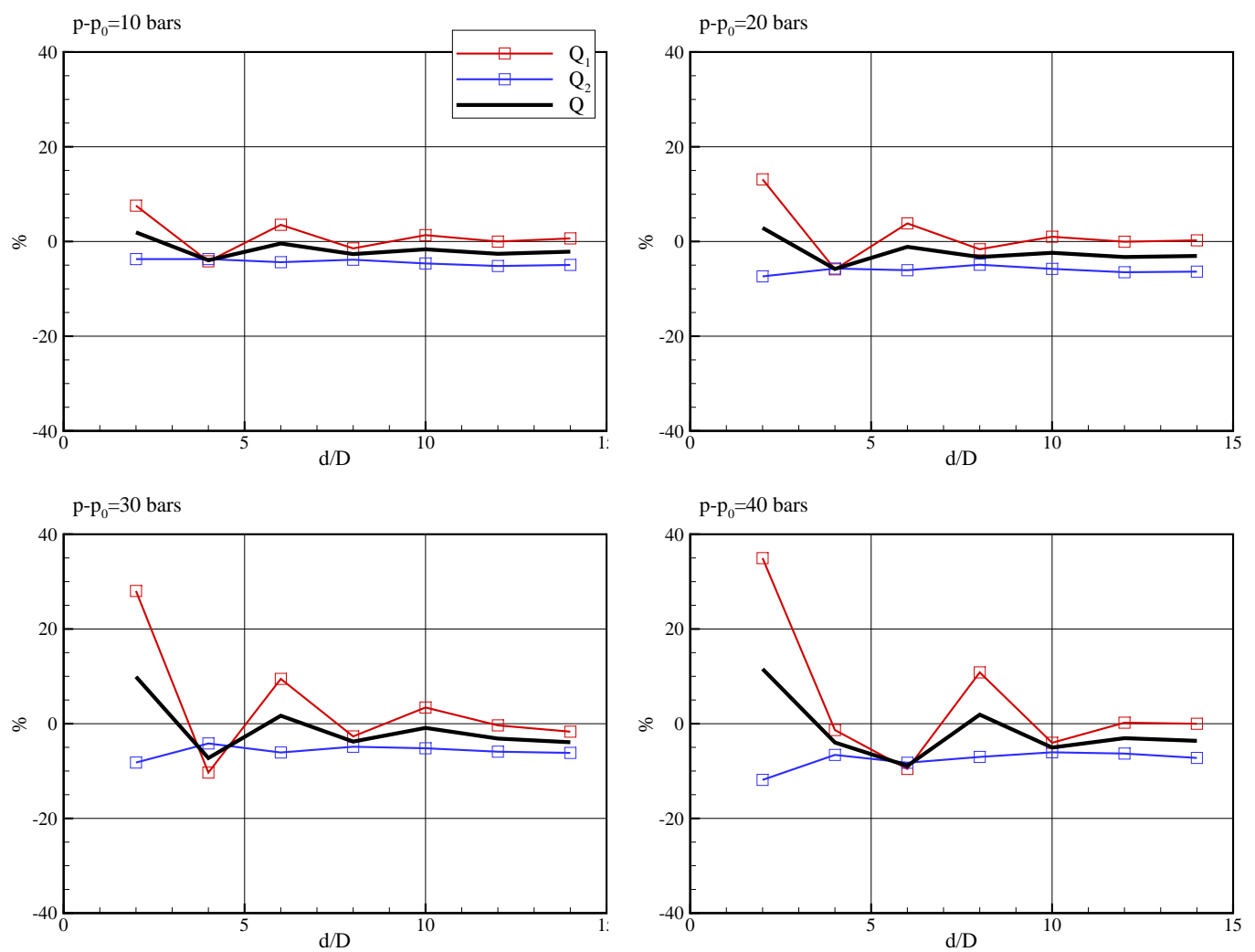

Figure 7: Perturbations of the energy absorption by wave interactions in an array of two systems as a function of their distance, in regular waves.
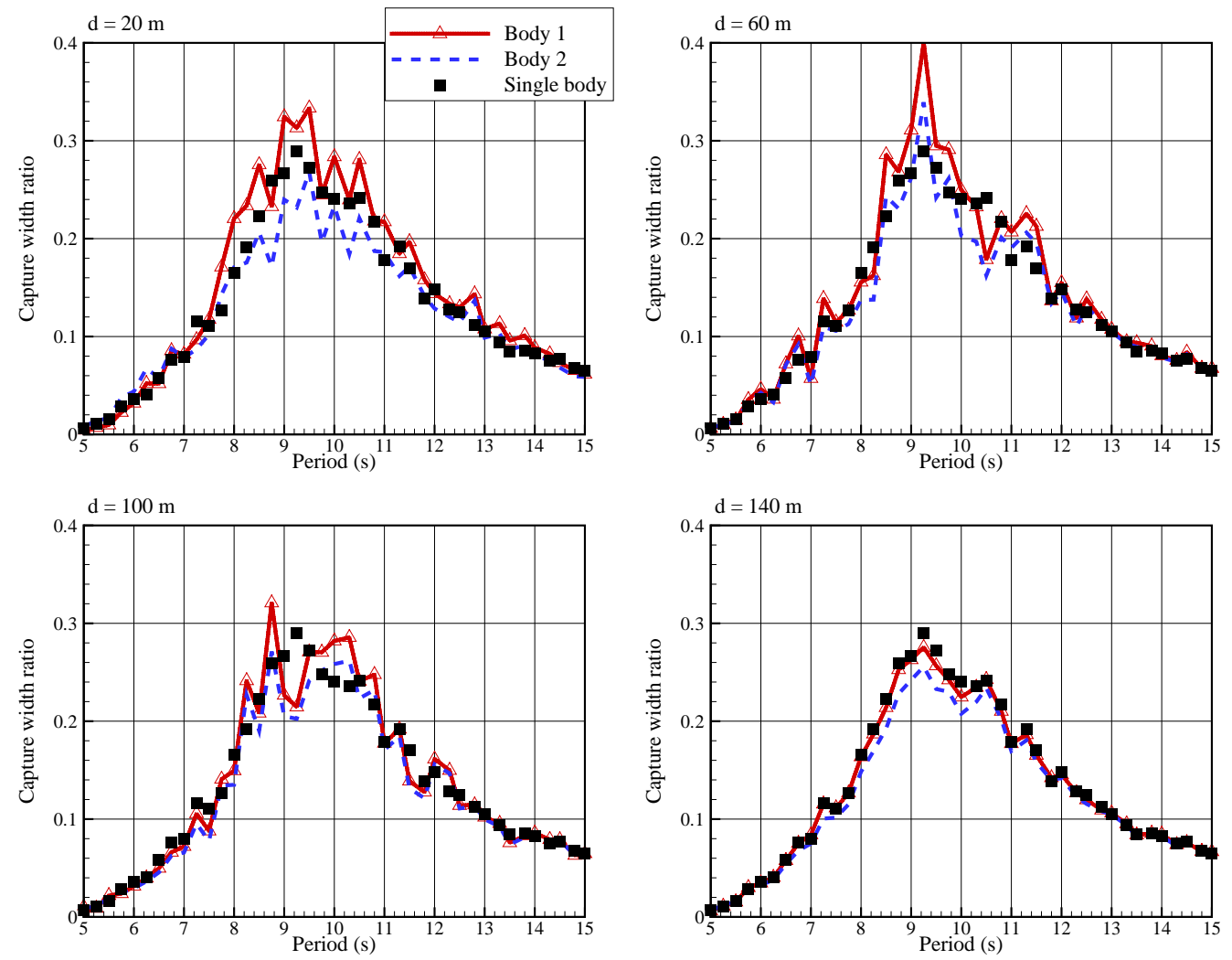

Figure 9: Capture width ratios of the first buoy and of the second buoy as a function of distance between them in irregular seas. In these simulations, $\Delta p=10$ bars and the wave amplitude was set equal to $2 \mathrm{~m}$. 
even when the distance between them is longer than 14 diameters.

- The wave interactions effects can lead to an increase in the energy absorption in only a few cases. Most of the time, the energy absorbed by the array is reduced in comparison with the one of isolated systems. In the cases of energy increase, it is due to a substantial increase of the energy absorbed by the front system, the energy absorbed by the second system being always reduced.

- When the systems are sufficiently separated, although noticeable, wave interactions effects are rather low (about a few \% of energy production).

- In the case of the generic heaving WECs, the trend of the wave interactions effect (positive/negative) does not seem to depend on the wave period and the same trends have been observed in regular and irregular waves. However, different results were found in the case of the SEAREV WEC.

However, the arrays we considered here are very basic. So, these results may not be the same when considering a more realistic array, composed of many more systems. This could have not been addressed in this paper, because state of the art potential theory based code are not numerically able to deal with many different systems, due to numerical limitations (particularly related with the storage of huge influence coefficients matrices). This problem could be solved using more advanced techniques, such as the Fast Multipole Method [23]. This topic is on-going research in Ecole Centrale de Nantes and will be published in a forthcoming paper.

\section{Acknowledgements}

This work has been supported by the Région Pays de la Loire and the Agence de l'Environnement et de la Maîtrise de l'Energie (ADEME) through the funding of the $\mathrm{PhD}$ studies of Bruno Borgarino.

\section{References}

[1] J. Tissandier, A. Babarit, and A.H. Clément. Study of the smoothing effect on the power production in an array of searev wave energy converters. In Proc. of the $18^{t} \mathrm{~h} \mathrm{In-}$ ternational Offshore and Polar Engineering Conference, ISOPE 2008, 2008.

[2] M. Molinas M., O. Skjervheim, B. Sorby, P. Andreasen, S. Lundberg, and T. Undeland. Power smoothing by aggregation of wave energy converters for minimizing electrical energy storage requirements. In Proc. of the 7 th European Wave and Tidal Energy Conference, 2007.

[3] K. Budal. Theory for absorption of wave power by a system of interacting bodies. Journal of Ship Researc, 21(4):248-253, 1977.

[4] D.V. Evans. A theory of wave absorption by oscillating bodies. J. Fluid Mechanics, 77:1-25, 1976.
[5] K. Budal and J. Falnes. Interacting point absorbers with controlled motion, in Power from Sea Waves. BM Count, Academic Press, 1980.

[6] G.P. Thomas and D.V. Evans. Arrays of threedimensional wave-energy absorbers. Journal of Fluid Mechanics, 108:67-88, 1981.

[7] M.J. Simon. Multiple scattering in arrays of axisymmetric wave-energy devices. part 1. a matrix method using a plane-wave approximation. Journal of Fluid Mechanics, 120:1-25, 1982.

[8] P. McIver and D.V. Evans. Approximation of wave forces on cylinder arrays. Applied Ocean Research, 6(101-107), 1984.

[9] A. F. de O. Falcão. Wave-power absorption by a periodic linear array of oscillating water columns. Ocean Engineering, 29:1163-1186, 2002.

[10] P.A.P. Justino and A.H. Clément. Hydrodynamic performance for small arrays of submerged spheres. In Proc. of the $5^{\text {th }}$ European Wave Energy Conference, Cork, Ireland, 2003.

[11] B. Child and V. Venugopal. Interaction of waves with an array of floating wave energy devices. In Proc. of the $7^{\text {th }}$ European Wave and Tidal Energy Conference, Porto, Portugal.

[12] Mícheál O’Catháin, Bernt J. Leira, and John V. Ringwood. Modelling of multibody marine systems with application to wave-energy devices. In Proc. of the $7^{\text {th }} \mathrm{Eu}$ ropean Wave and Tidal Energy Conference, Porto, Portugal, 2007.

[13] W.E. Cummins. The impulse response function and ship motions. Schiffstechnik, pages 491-502, 1962.

[14] B. King. Time-Domain Analysis of Wave Exciting Forces on Ships and Bodies. $\mathrm{PhD}$ thesis, The University of Michigan, 1987.

[15] B. Molin. Hydrodynamique des structures offshore. Guides Pratiques sur Les Ouvrages En Mer. TECHNIP, 2002.

[16] P. Ricci, J.B. Saulnier, A. F. de O. Falcão, and T. Pontes. Time-domain models and wave energy converters performance assessment. In Proc. of the ASME $27^{\text {th }}$ International Conference on Offshore Mechanics and Artic Engineering, Estoril, Portugal, 2008.

[17] A.H. Clément. Hydrodynamique instationnaire linéarisée : Mise en oeuvre d'une méthode de singularités utlisant un modèle différentiel de la fonction de Green. Technical Report LHN-9703, Laboratoire de Mécanique des Fluides de l'Ecole Centrale de Nantes, CNRS UMR 6598 , 1997.

[18] A. Babarit. Achil3D v2.0 : User Manual. Laboratoire de Mécanique des Fluides, CNRS UMR6598, Ecole Centrale de Nantes, October 2008.

[19] A.F. de O. Falcão. Modelling and control of oscillatingbody wave energy converters with hydraulic power takeoff and gas accumulator. Ocean Engineering, 34:20212032, 2007. 
[20] A. Babarit C. Josset and A.H. Clément. A wave-to-wire model for the searev wave energy converter. Proceedings of the Institution of Mechanical Engineers Part MJournal of Engineering for the Maritime Environment, 221(2):81-93, 2007.

[21] A. F. de O. Falcao. Phase control trough load control of oscillating-body wave energy converters with hydraulic pto system. Ocean Engineering, 35:35-366, 2008.
[22] S.H. Salter, J.RM. Taylor, and N.J. Caldwell. Power conversion mechanisms for wave energy. In Proc. of the Institution of Mechanical Engineers, Part M-Journal of Engineering for the Maritime Environment, volume 216, pages $1-27,2002$.

[23] L. Greengard and V. Rokhlin. A fast algorithm for particle simulations. Journal of Computational Physics, 73(2):325-348, 1987 